\title{
Correction to: A new approach for ergonomic risk assessment integrating KEMIRA, best-worst and MCDM methods
}

\author{
Elif Kılıç Delice ${ }^{1} \cdot$ Gülin Feryal Can ${ }^{2}$
}

Published online: 5 August 2020

(C) Springer-Verlag GmbH Germany, part of Springer Nature 2020

\section{Correction to: Soft Computing}

https://doi.org/10.1007/s00500-020-05143-9

Publisher's Note Springer Nature remains neutral with regard to jurisdictional claims in published maps and institutional affiliations.

Line 554, equation 11: $w_{x_{W_{k}}}$ must be written the equation (11) as below:

$\left|\frac{w_{x_{i_{k}}}}{w_{x_{W_{k}}}}-x_{i W_{k}}\right| \leq \xi_{k}^{x}$ for all $i$

Table 14: line $1, e_{r} j$ should be written as $e_{\mathrm{r}}$

Table 14 Weights of MOORA Ratio, MOORA reference point, MOOSRA and COPRAS methods

\begin{tabular}{llllll}
\hline Parameters & MOORA ratio & MOORA reference point & MOOSRA & COPRAS & SUM \\
\hline$e_{r}$ & 0.950 & 0.956 & 0.936 & 0.994 & \\
$1-e_{r}$ & 0.050 & 0.044 & 0.064 & 0.006 & 0.164 \\
$a w_{r}$ & 0.304 & 0.269 & 0.391 & 0.037 & \\
$\sqrt{a w_{r}}$ & 0.551 & 0.518 & 0.625 & 0.192 & \\
$1+\sqrt{a w_{r}}$ & 1.551 & 1.518 & 1.625 & 1.192 & 5.886 \\
$p w_{r}$ & 0.263 & 0.258 & 0.276 & 0.202 & 1.000 \\
\hline
\end{tabular}

The original article can be found online at https:// doi.org/10.1007/s00500-020-05143-9.

$\square$ Elif Kılıç Delice

elif.kdelice@atauni.edu.tr

1 Industrial Department, Atatürk University, Erzurum, Turkey

2 Industrial Department, Baskent University, Erzurum, Turkey 